

\title{
Light irradiation of matter in neutron scattering experiments
}

\author{
A. Stöckli, A. Furrer
}

\section{To cite this version:}

A. Stöckli, A. Furrer. Light irradiation of matter in neutron scattering experiments. Revue de Physique Appliquée, 1984, 19 (9), pp.751-754. 10.1051/rphysap:01984001909075100 . jpa-00245252

\section{HAL Id: jpa-00245252 https://hal.science/jpa-00245252}

Submitted on 1 Jan 1984

HAL is a multi-disciplinary open access archive for the deposit and dissemination of scientific research documents, whether they are published or not. The documents may come from teaching and research institutions in France or abroad, or from public or private research centers.
L'archive ouverte pluridisciplinaire HAL, est destinée au dépôt et à la diffusion de documents scientifiques de niveau recherche, publiés ou non, émanant des établissements d'enseignement et de recherche français ou étrangers, des laboratoires publics ou privés. 


\title{
Light irradiation of matter in neutron scattering experiments
}

\author{
A. Stöckli and A. Furrer \\ Institut für Reaktortechnik, ETH Zürich, CH-5303 Würenlingen, Switzerland
}

\begin{abstract}
Résumé. - Nous avons construit un cryostat pour effectuer simultanément la diffusion des neutrons et l'absorption des photons. La source des phonons est une lampe halogène, de larges bandes de longueurs d'onde entre $400 \mathrm{~nm}$ et $1000 \mathrm{~nm}$ sont sélectionnées au moyen des filtres appropriés. La lumière est guidée par un système de lentilles jusqu'à l'échantillon monté dans le cryostat. La lumière peut être interrompue périodiquement par un système mécanique qui fonctionne à des fréquences entre $0,05 \mathrm{~Hz}$ et $5 \mathrm{~Hz}$. Le nombre des neutrons diffusés pendant les expériences obscures et éclairées est accumulé séparément. Nous avons fait usage de notre appareil pour étudier au moyen de la diffusion inélastique des neutrons la dynamique des systèmes de chlorophylle photosynthétique induite par des photons.
\end{abstract}

\begin{abstract}
We have developed a device for performing neutron scattering experiments with simultaneous irradiation by light at low temperatures. The light source is a halogen lamp which yields broad bands of wavelengths between $400 \mathrm{~nm}$ and $1000 \mathrm{~nm}$ by using appropriate filters. The light is guided by a series of lenses to the sample which is mounted in a cooling system. A mechanical chopper may be inserted into the light beam in order to create light pulses with frequencies between $0.05 \mathrm{~Hz}$ and $5 \mathrm{~Hz}$, and the neutron counts resulting from the dark and light experiments are separately stored. Our device has been used to study the light-induced dynamical behaviour of photosynthetic chlorophyll systems by neutron spectroscopy.
\end{abstract}

\section{Introduction.}

When an optically active system is illuminated by light of a particular wavelength, it is electronically excited. After some time the excited system will return to its ground state. The corresponding relaxation mechanism can be either radiative, resulting in the emission of a photon (fluorescence), or radiationless, in which case a number of vibrational or phonon modes are created. Relaxation mechanisms generally take place through the simultaneous occurrence of both radiative and radiationless processes subject to energy conservation. Radiationless transitions increase the occupation number of the phonons and vibrons which can be directly measured by inelastic neutron scattering (INS). The INS technique appears to be the only experimental method which provides direct information on the radiationless transitions in terms of elementary quantum-mechanical processes, i.e. on the selection rules and lifetimes of the phonons and vibrons in the electronic-vibrational relaxation mechanisms.

The present paper describes how simultaneous photon absorption and neutron scattering can be experimentally realized at low temperatures. An ins- trumental device has been developed which is outlined in detail in section 2. An important aspect of the instrumental setup appears to be the possibility to create light pulses which is useful to unambiguously determine the amount of heat dissipated into the system under light irradiation. In section 3 we present some preliminary results of INS experiments performed for a chlorophyll-protein system in $\mathrm{D}_{2} \mathrm{O}$ solution. Some conclusions are summarized in section 4.

\section{Instrumental configuration.}

Figure 1 shows the instrumental configuration realized at our institute. The light source is a $100 \mathrm{~W}$ halogen lamp whose efficiency is optimized by using a reflector. A parallel beam of $5 \mathrm{~cm}$ diameter is created after transmission through a plane-convex lens. Various broad-band filters of variable band width have been used to yield wavelengths between $400 \mathrm{~nm}$ and $1000 \mathrm{~nm}$. The light is guided to the sample through a lens system whose focal distance can be adjusted to the sample geometry. The sample is mounted in a closed-cycle $\mathrm{He}$ refrigerator. The light beam penetrates the sample chamber through a glass disk or lens inserted into the radiation shield of the cooling system. 


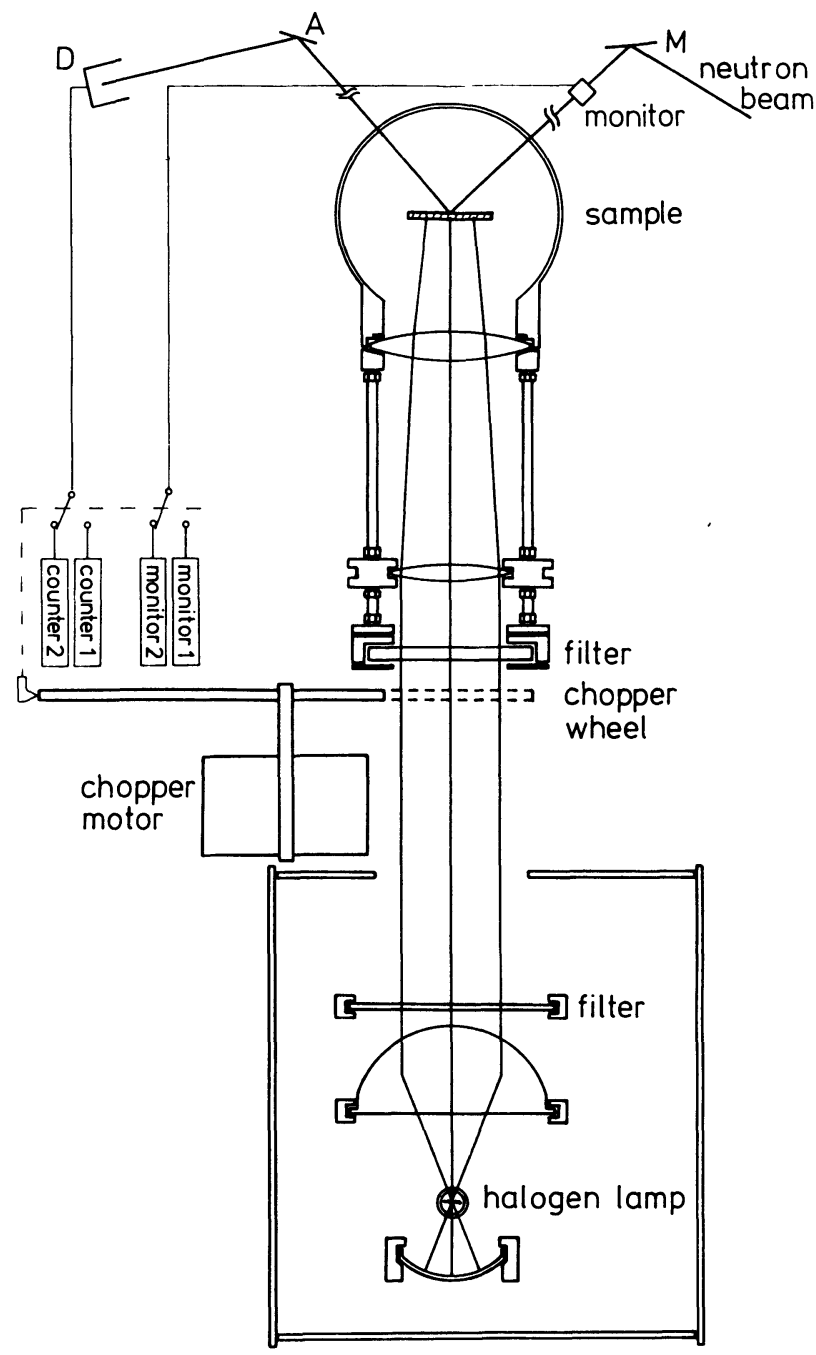

Fig. 1. - Conceptual design of the instrumental device developed for simultaneous light irradiation and neutron scattering experiments at low temperatures.

The maximum intensity at the sample position is 2 MLux.

In order to be able to unambiguously discriminate between heating effects and actual nonequilibrium phenomena in the INS experiments, a mechanical chopper has been inserted into the light beam. The light chopper is a rotating wheel with regularly arranged openings which periodically interrupt the light beam, thus creating light pulses whose frequencies can be varied between $0.05 \mathrm{~Hz}$ and $5 \mathrm{~Hz}$. The neutron count-rates are separately stored for the dark and light experiments.

A crucial part of the instrumental setup is the sample cell which has to meet simultaneously three different experimental requirements, namely optimum scattering properties in the INS experiments, optimum efficiency concerning light irradiation and optimum cooling. Figure 2 schematically shows the sample container used in our experiments which is particularly suited for solutions. The actual sample geometry

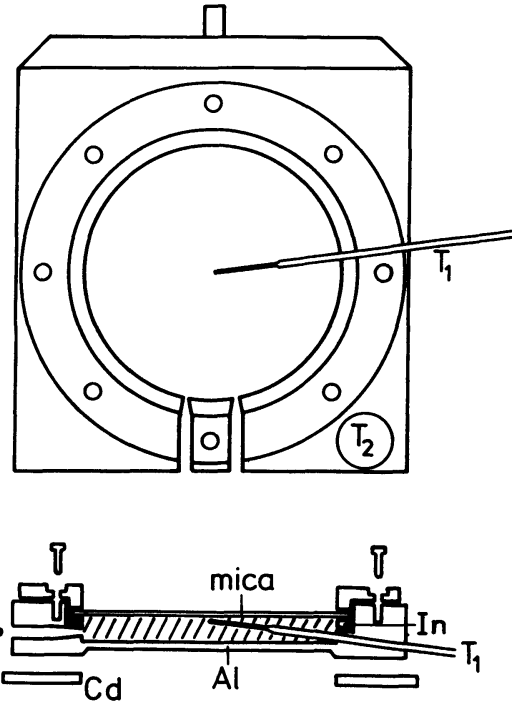

Fig. 2. - Schematical ground-plan and vertical section of the sample cell.

corresponds to a cylinder of $2.5 \mathrm{~cm}$ diameter and $0.15 \mathrm{~cm}$ height. The bottom window is made of aluminium which is transparent for neutrons and guarantees a good heat conduction as well. The top part consists of an optically transparent mica window of $0.07 \mathrm{~cm}$ thickness. When the solution freezes in the cooling process, the mica window does not break because of its high elasticity. An indium ring is used to seal the mica window with the bottom part. The solution is injected into the sample cell through a small hole at the circumference of the cylinder. The temperature is measured in the centre of the sample $\left(T_{1}\right)$ as well as at the sample frame $\left(T_{2}\right)$ in order to determine the temperature gradient usually set up under light irradiation.

In the instrumental setup described above the light plane coincides with the neutron scattering plane, thus the INS experiments can only be performed in the reflectivity configuration as indicated in figure 1. This configuration is ideal when the absorption of both light and neutrons in the sample is large. For the case of small light and neutron absorption the INS experiments may more easily be carried out in the transmission configuration, since the light plane can then be chosen perpendicular to the neutron scattering plane. A sketch of the sample cell realized in the course of our experiments is shown in figure 3.

\section{Experimental results for photosynthetic chlorophyll systems.}

The function of photosynthetic systems is to absorb light energy and to convert it into chemical energy with optimum efficiency. The energy transfer mechanism occurs through several steps, e.g. energy transfer from the electronically excited antenna chlorophyll complexes to the reaction centre. Part of the 


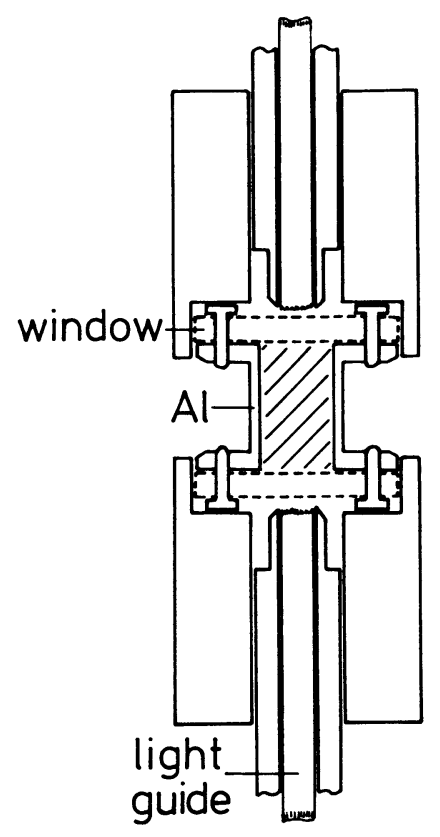

Fig. 3. - Schematical sketch of the sample cell.

excitation energy, however, may be lost because of the electronic-vibrational coupling in which case low energy phonons are created. This effect increases the phonon occupation number $n(\omega)$ which can be directly measured by INS-experiments through the incoherent cross section

$$
\begin{aligned}
\left(\frac{\mathrm{d}^{2} \sigma}{\mathrm{d} \Omega \mathrm{d} \omega}\right)_{\mathrm{inc}} \sim \frac{k_{1}}{k_{0}} \frac{\sigma_{\mathrm{inc}}}{M} Q^{2} \mathrm{e}^{-2 W(Q)} \frac{g(\omega)}{\omega} \times \\
\times\left\langle\frac{1}{2} \pm \frac{1}{2}+n(\omega)\right\rangle .
\end{aligned}
$$

We have performed INS experiments for various chlorophyll systems in solution. As an example we present the results obtained for a suspension of membranes of the photosynthetic bacterium Rhodopseudomonas viridis in $\mathrm{D}_{2} \mathrm{O}$ buffer at a protein concentration of roughly $20 \mathrm{mg} / \mathrm{ml} \mathrm{[1].} \mathrm{The} \mathrm{effect}$ of light irradiation $(400 \leqslant \lambda \leqslant 800 \mathrm{~nm})$ is shown in the normalized energy spectra of figure 4 . There is a considerable increase of the inelastic scattering upon illumination. The light effect appears to be symmetric with respect to the energy-gain and energy-loss part of the spectrum. It is evident from equation (1) that this can only be explained by increasing the phonon occupation number from $n(\omega)$ to $n^{*}(\omega)$, whereas the density-of-states $g(\omega)$ remains unaltered. It should also be noted that a temperature gradient was set up under light irradiation $\left(T_{1}=55 \mathrm{~K}\right.$ for the centre of the sample, $T_{2}=28 \mathrm{~K}$ for the sample frame), whereas for the dark experiment the sample temperature was kept constant at $28 \mathrm{~K}$.

During illumination there is a continuous production of phonons, so that the phonon system is not in thermal equilibrium. Consequently the occupation

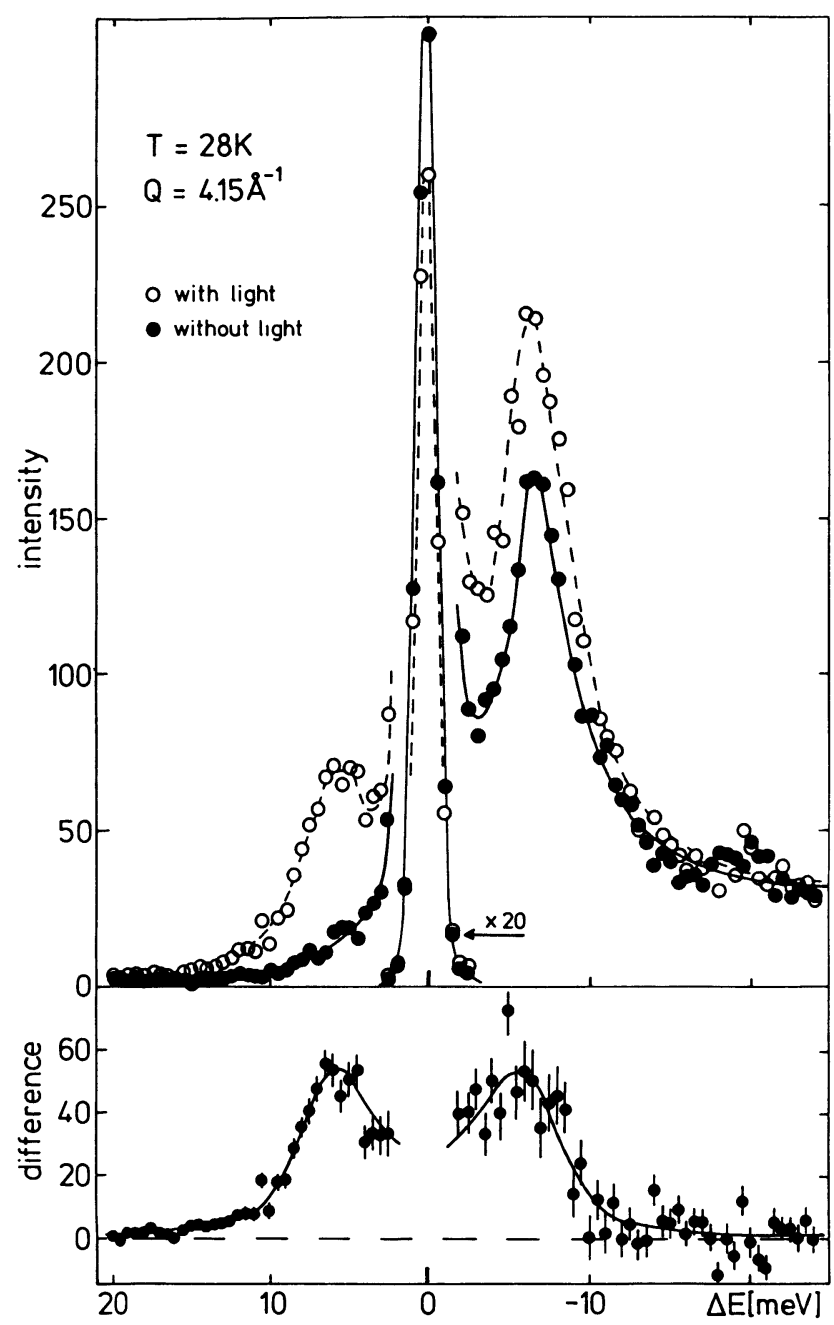

Fig. 4. - Normalized energy spectra of neutrons scattered from a suspension of membranes of the photosynthetic bacterium Rhodopseudomonas viridis in $\mathrm{D}_{2} \mathrm{O}$ buffer. The lines are drawn as guides to the eye.

number $n(\omega)$ is not governed by Bose-Einstein statistics. Anharmonic effects tend to drive the system back into thermal equilibrium. The two competing effects may be described by a modified Boltzmann equation

$$
\frac{\mathrm{d} \Delta n(\omega)}{\mathrm{d} t}=-\frac{\Delta n(\omega)}{\tau(\omega)}+I(\omega),
$$

where $I(\omega)$ is the number of phonons produced under light irradiation, $\tau(\omega)$ the mean phonon lifetime and $\Delta n(\omega)$ the deviation of the occupation number $n(\omega)$ from Bose-Einstein statistics. In the steady state the solution of equation (2) is

$$
\Delta n(\omega)=I(\omega) \tau(\omega) .
$$

For $\Delta n(\omega) \ll 1$ the system is not driven much out of equilibrium, so that the occupation number $n^{*}(\omega)$ can be explained in terms of a heat effect with the system being in thermal equilibrium at a temperature 
$T^{*}>T$. Using equation (1) we have calculated $n^{*}(\omega)$ from the observed energy spectra and determined $T^{*}$ to be $\sim 75 \mathrm{~K}$ which is considerably higher than the value $T_{1}=55 \mathrm{~K}$ measured in the centre of the sample. This means that we have really investigated a non-equilibrium system with $\Delta n(\omega) \simeq 1$ for $\hbar \omega<10 \mathrm{meV}$. Obviously the anharmonic phonon coupling was not strong enough to stabilize the system in thermal equilibrium. At present we are performing an experiment in which the light beam is mechanically pulsed, so that our hypothesis can be unambiguously checked.

\section{Conclusion.}

An instrumental setup has been described which allows simultaneous photon absorption and neutron scattering experiments at low temperatures. A theoretical account of combined neutron-photon processes in condensed matter has recently been published [2]. In our case the light source is a halogen lamp, but it may sometimes be advantageous to use laser light which has an almost infinitely narrow band width and generally more intensity, particularly when a pulsed laser is used. In addition laser light offers cryotechnical advantages, since the laser beam can penetrate the inside of a cryostat through a rather small hole in the radiation shield, so that low temperatures can more easily be stabilized. Moreover the laser technique can be applied to INS experiments in both reflection and transmission configuration by using a suitable system of mirrors and lenses in the inside of the cryostat.

\section{Acknowledgments.}

The expert technical assistance of M. Koch is gratefully acknowledged.

\section{References}

[1] Furrer, A., Stöckli, A., Hälg, W., Kühlbrandt, W., Mühlethaler, K. and Wehrli, E., Helv. Phys. Acta 56 (1983) 655.

[2] Lovesey, S. W. and Meech, G. P., Z. Phys. B 51 (1983) 99. 